Article

\title{
Function of SREBP1 in the Milk Fat Synthesis of Dairy Cow Mammary Epithelial Cells
}

\section{Nan Li, Feng Zhao, Chenjie Wei, Mengyao Liang, Na Zhang, Chunmei Wang, Qing-Zhang Li and Xue-Jun Gao *}

Key Laboratory of Agricultural Biological Functional Genes, Northeast Agricultural University, Harbin 150030, China; E-Mails: linan0829@163.com (N.L.); erjinzhi@126.com (F.Z.); chengjiewin@163.com (Che.W.); liangmengyao2008@sina.com (M.L.); nazhang1981@126.com (N.Z.); wangcm-1@sohu.com (Chu.W.); qzli@neau.edu.cn (Q.-Z.L.)

* Author to whom correspondence should be addressed; E-Mail: gaoxj5390@sina.com; Tel.: +86-451-5519-0244; Fax: +86-451-5519-0244.

Received: 6 July 2014; in revised form: 23 August 2014 / Accepted: 4 September 2014 / Published: 23 September 2014

\begin{abstract}
Sterol regulatory element-binding proteins (SREBPs) belong to a family of nuclear transcription factors. The question of which is the most important positive regulator in milk fat synthesis in dairy cow mammary epithelial cells (DCMECs) between SREBPs or other nuclear transcription factors, such as peroxisome proliferator-activated receptor $\gamma$ (PPAR $\gamma$ ), remains a controversial one. Recent studies have found that mTORC1 (the mammalian target of rapamycin $\mathrm{C} 1$ ) regulates SREBP1 to promote fat synthesis. Thus far, however, the interaction between the SREBP1 and mTOR (the mammalian target of rapamycin) pathways in the regulation of milk fat synthesis remains poorly understood. This study aimed to identify the function of SREBP1 in milk fat synthesis and to characterize the relationship between SREBP1 and mTOR in DCMECs. The effects of SREBP1 overexpression and gene silencing on milk fat synthesis and the effects of stearic acid and serum on SREBP1 expression in the upregulation of milk fat synthesis were investigated in DCMECs using immunostaining, Western blotting, real-time quantitative PCR, lipid droplet staining, and detection kits for triglyceride content. SREBP1 was found to be a positive regulator of milk fat synthesis and was shown to be regulated by stearic acid and serum. These findings indicate that SREBP1 is the key positive regulator in milk fat synthesis.
\end{abstract}

Keywords: SREBPs; mTOR; dairy cow mammary epithelial cells; milk fat synthesis 


\section{Introduction}

Sterol regulatory element-binding proteins (SREBPs) belong to the basic helix-loop-helix-leucine zipper (bHLH-Zip) family of transcription factors, which are synthesized as inactive precursors bound to the endoplasmic reticulum (ER). SREBP precursors exit the ER and transit to the Golgi apparatus, where two distinct proteases cleave the SREBP precursor to release the transcriptionally active $N$-terminus. The $N$-terminus contains the bHLH-Zip region that, upon binding to DNA, functions as a transcription factor. Each SREBP precursor exists in a hairpin-like conformation of $\sim 1150$ amino acids $(\sim 125 \mathrm{kDa})$ organized into three domains: an $\mathrm{NH}_{2}$-terminal cytoplasmic domain that functions as a DNA-binding transcription factor; a central domain containing two transmembrane segments projecting into the lumen of the ER; and a $\mathrm{COOH}$-terminal cytoplasmic domain that performs essential regulatory functions. The mammalian genome encodes three SREBP isoforms designated SREBP1a, SREBP1c, and SREBP2. SREBP1a and SREBP1c are encoded by the same gene (SREBP1), which is regulated by two distinct promoters and alternative splicing [1]. The original cloning of the SREBP1 gene from human HeLa cells yielded several partial cDNA clones with alternative sequences at both the $5^{\prime}$ and $3^{\prime}$ ends, which were postulated to result from alternative splicing. SREBPla and SREBPIc differ in sequence at both their 5' and 3' ends. SREBPla consists of exons la, 18a, and 19a, while SREBP1c consists of exons 1c, 18c, and 19c. Exons la and lc are separated by $\sim 14 \mathrm{~kb}$ [2]. The two different sequences at each end were designated "a" and "c". The full-length human cDNA containing "c" sequences at both ends was designated SREBPIC and the full-length cDNA isolated from Chinese hamster ovary $(\mathrm{CHO})$ cells contained "a" sequences at both ends was designated SREBPIa [3]. The $N$-terminus contains a DNA activation domain which can bind transcriptional coactivators, such as CBP [4]. SREBP1 activates mainly genes encoding fatty acid (FA) and triglyceride biosynthesis: SREBP1a activates the genes required in the synthesis of cholesterol and fatty acid, such as fatty acid elongase and stearoyl-CoA desaturase, while SREBP1c directly activates the expression of more than 30 genes dedicated to the synthesis and uptake of fatty acids and triglycerides. The target genes of SREBP1c include low density lipoprotein (LDL) receptor, ACC (acetyl-CoA carboxylase), FAS (fatty acid synthase), SCD (stearoyl-CoA desaturase), INSIG1 (insulin induced gene-1), S14, GK (glucokinase), and PEPCK (phosphoenolpyruvate carboxykinase), which are involved in milk fat synthesis and glucose synthesis [5,6]. The target genes of SREBP2 include HMGCS (3-hydroxy-3-methylglutarylcoenzyme a synthase), HMGCR (HMGC reductase), FDPS (farnesyl diphosphate synthase), SS (squalene synthase), and DNAJA4 (DNAJ (HSP40) homolog subfamily-A member-4) [7,8], which are involved in either the cholesterol biosynthesis pathway or in cholesterologenesis. When cells (such as liver cells) are stimulated by oxygen sterol, LXR (liver X receptor), NF-Y (nuclear factor-Y), and Spl (specificity protein 1) signal, SREBPs are activated. The inactive precursors are activated via a splicing system (SCAP, S1P, and S2P) consisting of a two-step photolytic cleavage by site-1 (S1P) and site-2 (S2P). Following cleavage, the amino-terminal fragments of SREBPs (nSREBP1c) translocate to the nucleus as homodimers and bind to sterol regulatory elements (SREs) within the promoters of target genes [9].

Ma and Corl found that SREBP1 plays an important role in the integrated regulation of lipid synthesis in dairy cow mammary epithelial cells (DCMECs) through regulation of key enzymes [10]. Milk fat is composed predominantly of triglycerides containing fatty acids that arise from two sources: 
de novo synthesis within the mammary gland and the uptake of long-chain fatty acids from circulation. SREBP1 regulates lipid synthesis in DCMECs by controlling the transcription of genes encoding enzymes involved in de novo FA synthesis, FA desaturation, long-chain FA uptake, and triglyceride esterification. Recent findings reported by Loor et al. [11] challenged the proposal that SREBP1 is central for milk fat synthesis regulation and highlight a pivotal role for a concerted action among PPARG, PPARGC1A, and INSIG1. Wan showed that the active compound in Vaccaria segetalis has similar functions as estrogen and/or prolactin in DCMECs, increasing the expressions of prlr, ero, akt1, and elf5 genes, while repressing peroxisome proliferater-activated receptor (PPAR $\gamma)$ expression [12]. Findings reported by Huang indicate that SOCS3 acts as an inhibitor of the JAK2/STAT5a (janus kinase2/signal transducer and activator of transcription 5a) pathway and disturbs FA synthesis by decreasing SREBP1c expression, thus validating its involvement in fat synthesis [13]. Liu showed the liver expression of lipogenic genes including SREBPIC (560\%), FAS (190\%), ACC (48\%), and SCD1 (286\%) to be elevated by ethanol. Luteolin was shown to reduce the ethanol-induced expression of these genes in the liver: SREBP1c (79\%), FAS (80\%), ACC (60\%), and SCD1 (89\%) in mice hepatocytes. Furthermore, the ethanol-induced reduction of AMP-activated protein kinase and SREBP1c phosphorylation was shown to be abrogated by luteolin [14]. From the above results it is clear that there is still controversy regarding the question of which is the most important positive regulator in milk fat synthesis in DCMECs between SREBPs or other nuclear transcription factors, such as PPAR $\gamma$.

A role for $\mathrm{mTOR}$, the mammalian target of rapamycin, in promoting protein synthesis has been well described, and SREBP is known to play an important role in regulating lipid synthesis [15]. Studies have shown that insulin drives hepatic lipogenesis by inducing SREBP1c, and the inhibition of mTORC1 by rapamycin has been shown to dramatically reduce the expression of SREBP1c in vitro and in vivo, indicating that $\mathrm{mTORC1}$ plays an important role in controlling SREBP1c expression [16-18]. Previous work has also shown that mTORC1 positively regulates the activity of SREBP1 and that the mTOR pathway regulates several anabolic and catabolic pathways at the mRNA expression level [18-20]. However, rapamycin does not affect SREBP target gene expression in all cellular contexts [15,21]. It was recently demonstrated that mTORC1 regulates SREBP by controlling the nuclear entry of lipin 1, a phosphatidic acid phosphatase [22]. Although there are some reports about the role of SREBP1c in mouse and human, the molecular events associated with the regulation of milk fat synthesis in DCMECs remain largely unknown. The aim of this study was to investigate the functional role of SREBP1 in milk fat synthesis in DCMECs and to determine whether SREBP1 interacts with the mTOR pathway to regulate milk fat synthesis.

\section{Results and Discussion}

2.1. Sterol Regulatory Element-Binding Protein 1 (SREBP1) Overexpression in Dairy Cow Mammary Epithelial Cells (DCMECs) Increases the Expression of Lipogenic Genes and Key Enzymes of Fatty Acid Synthesis as Well as Triglyceride Secretion

The mRNA expression of SREBP1, ACC, FAS, SCD, m-TOR, and FABP3 (fatty acid-binding protein) was significantly increased in the pGCMV-IRES-EGFP-SREBP1 group compared with 
the empty vector group (Figure 1A), whereas PPAR $\gamma$ was found to be downregulated. The protein expression levels of SREBP1, p-SREBP1, mTOR, and p-mTOR were notably increased in cells transfected with SREBP1 compared with cells in the empty vector group (Figure 1B,C). Overexpression of SREBP1 in DCMECs was found to significantly increase triglyceride secretion (Figure 1D). These findings reveal that the overexpression of SREBP1 increases milk fat synthesis in DCMECs.

Figure 1. Effect of sterol regulatory element-binding protein 1 (SREBP1) overexpression on milk fat synthesis in dairy cow mammary epithelial cells (DCMECs). Three groups of DCMECs were assessed: nontransfected group, pGCMV-IRES-EGFP empty vector group (control group) and pGCMV-IRES-EGFP-SREBP1 group. (A) Relative mRNA levels (gene of interest/ $\beta$-actin) of indicated genes were determined by qRT-PCR after gene overexpression of SREBP1; (B) Expression levels of indicated proteins were assessed by Western blotting. $\mathrm{P}$ and $\mathrm{N}$ denote the precursor and cleaved nuclear forms of SREBP1, respectively; (C) Quantification of protein of interest/ $\beta$-actin relative fold by gray scale scan; and (D) Triglyceride (TG) content in the culture supernatant of DCMECs. Values represent means $\pm \mathrm{SE}$ (standard error) $(n=3)$. ${ }^{*} p<0.05, * * p<0.01$ compared with the pGCMV-IRES-EGFP empty vector group.

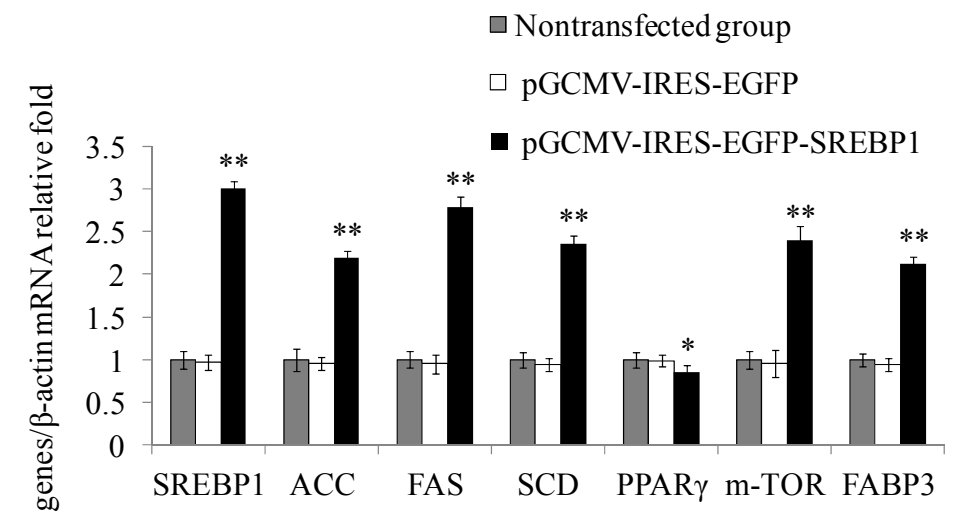

(A)

$\square$ Nontransfected group

$\square$ pGCMV-IRES-EGFP

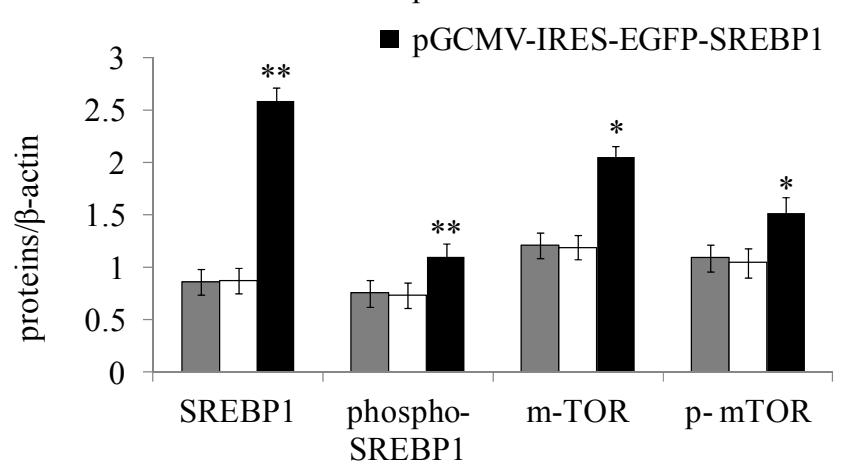

(C)

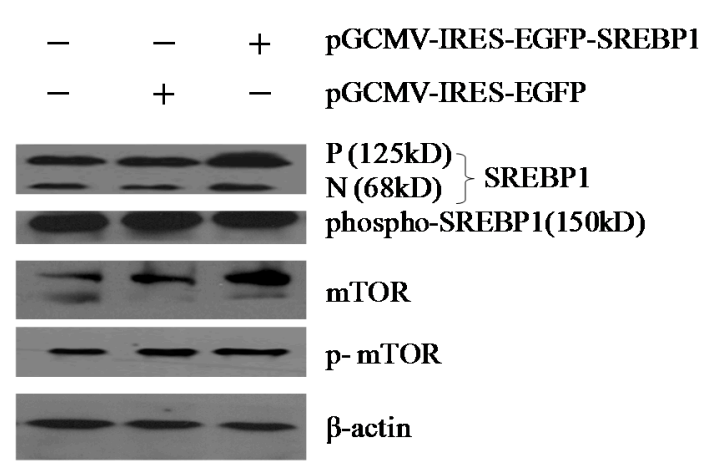

(B)

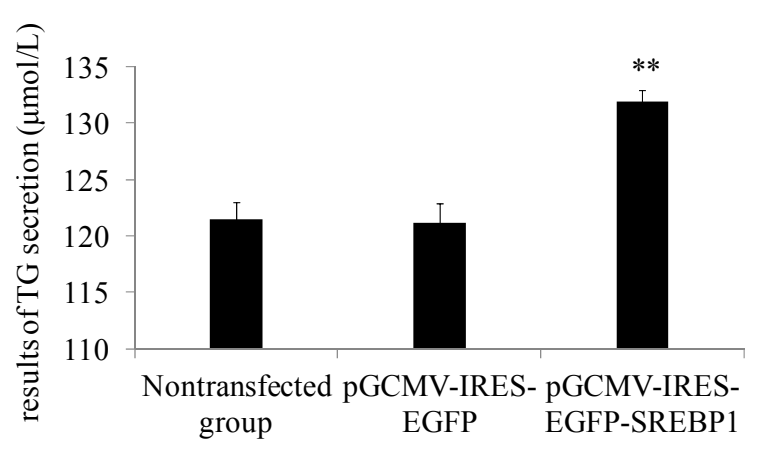

(D) 
2.2. SREBP1 Gene Silencing Decreases the Expression of Lipogenic Genes and Key Enzymes of Fatty Acid Synthesis and Decreases Triglyceride Secretion in DCMECs

SREBP1 knockdown by siRNA transfection was verified using qRT-PCR. SREBP1 gene silencing decreased the mRNA expression levels of SREBP1, ACC, FAS, SCD, mTOR, and FABP3 in cells transfected with $S R E B P 1$ siRNA compared with cells transfected with the empty vector, while PPAR was up-regulated in SREBP1 siRNA-transfected cells (Figure 2A). The protein expression levels of SREBP1, p-SREBP1, mTOR, and p-mTOR were notably decreased in cells transfected with SREBP1 siRNA compared with cells in the negative control group (Figure 2B,C). SREBP1 gene silencing in DCMECs was further found to result in significantly decreased triglyceride secretion (Figure 2D). These findings reveal that gene silencing of SREBPI decreases milk fat synthesis in DCMECs.

Figure 2. Effect of $S R E B P 1$ gene silencing on milk fat synthesis in DCMECs. Three groups of DCMECs were assessed: nontransfected group, negative group (control group), SREBP1 siRNA-transfected group. (A) Relative mRNA levels (gene of interest/ $\beta$-actin) of indicated genes were determined by qRT-PCR after gene silencing of SREBP1; (B) Expression levels of indicated proteins were assessed by Western blotting. P and $\mathrm{N}$ denote the precursor and cleaved nuclear forms of SREBP1, respectively; (C) Quantification of protein of interest/ $\beta$-actin relative fold by gray scale scan; and (D) TG content in the culture supernatant of DCMECs. Values represent means $\pm \operatorname{SE}(n=3)$. * $p<0.05$, ** $p<0.01$ compared with negative control group.

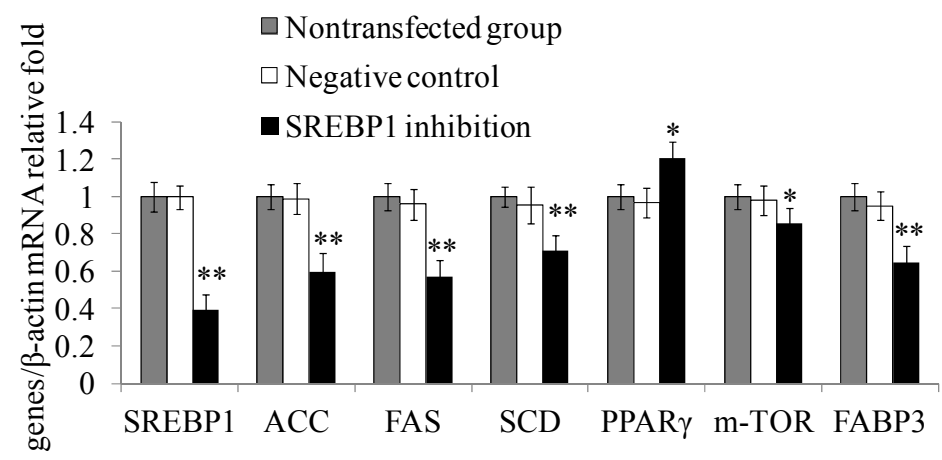

(A)

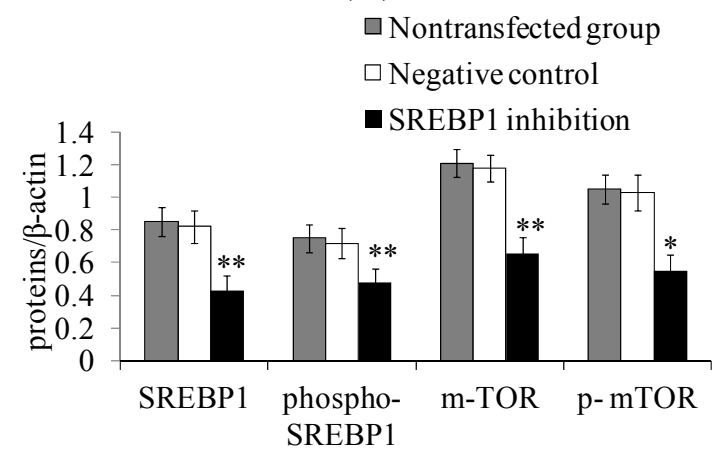

(C)

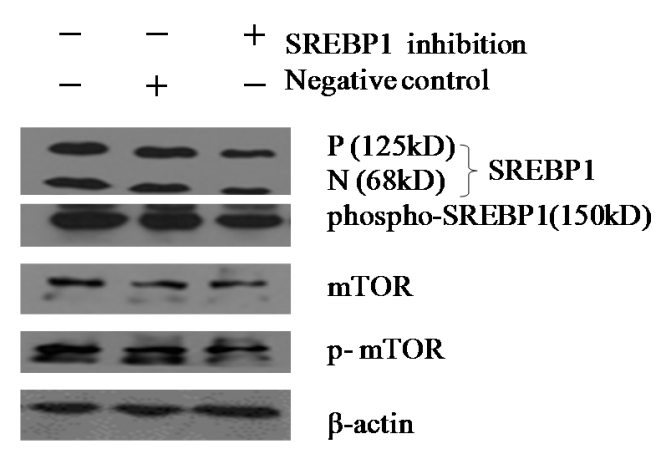

(B)

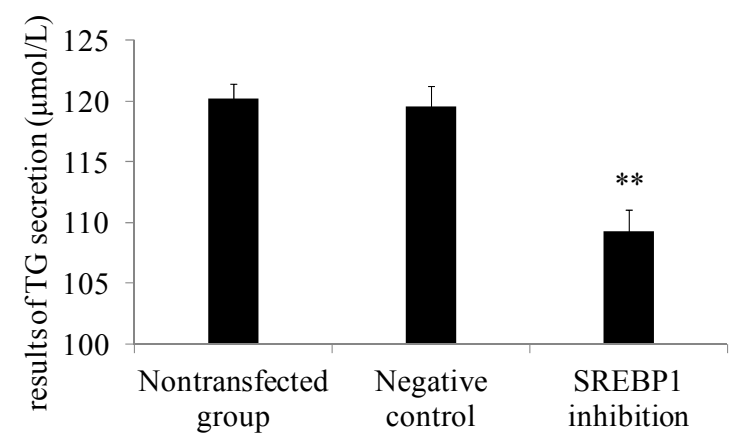

(D)

Lower expression levels of SREBP1 (Figure 3A,B) and p-mTOR (Figure 3C,D) in response to $S R E B P 1$ gene silencing were also demonstrated by immunofluorescence microscopy. SREBPl gene silencing in DCMECs was further found to result in significantly decreased lipid droplet content 
(Figure 3E). These results are in accord with the aforementioned conclusion, further revealing SREBP1 as a key positive regulator in milk fat synthesis in DCMECs.

Figure 3. Effect of SREBP1 gene silencing on the subcellular localization of molecules related to milk fat synthesis in DCMECs. Groups of DCMECs were assessed as in Figure 2. (A) Localization and integral optical density analysis of SREBP1 and p-mTOR. SREBP1 and p-mTOR (green), giantin (purple), and DAPI (blue). Scale bars $=10 \mu \mathrm{m}$; (B) Integral optical density analysis of SREBP1 and p-mTOR. p-mTOR (green), giantin (purple), and DAPI (blue). Scale bars $=10 \mu \mathrm{m}$; and $(\mathbf{C}, \mathbf{D})$ Immunofluorescent staining and integral optical density analysis of lipid droplets were performed on DCMECs. Lipid droplet (green) and DAPI (blue). Scale bars $=10$ or $15 \mu \mathrm{m}$. Values represent means $\pm \operatorname{SE}(n=3)$. ** $p<0.01$ compared with negative control group.

Nontransfected Negative control SREBP1 inhibition
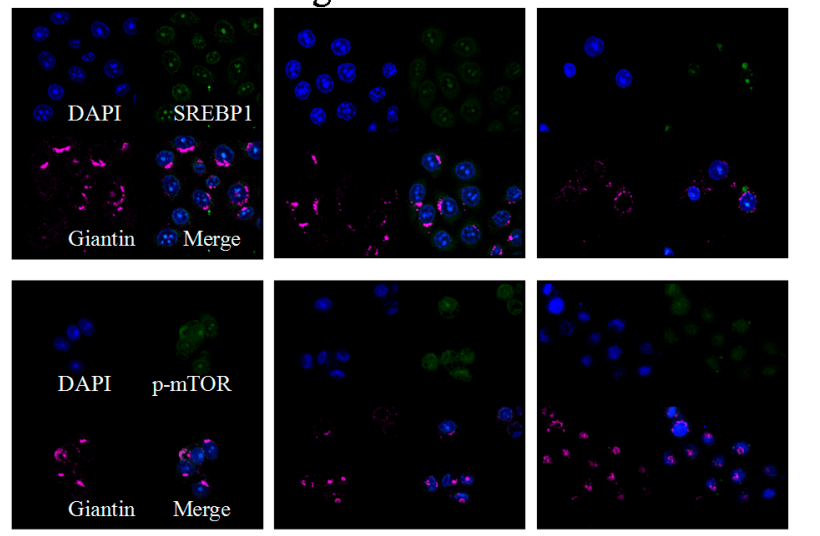

(A)
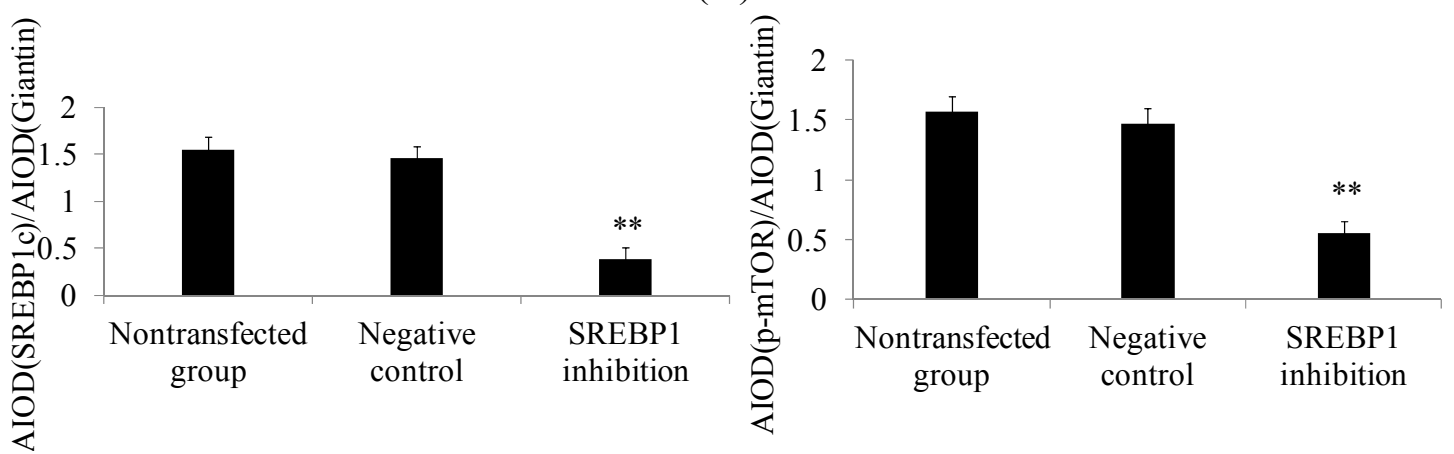

(B)

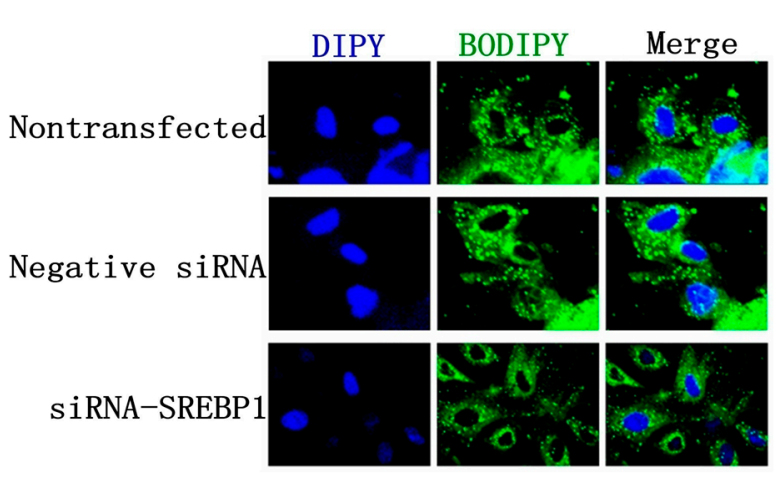

(C)

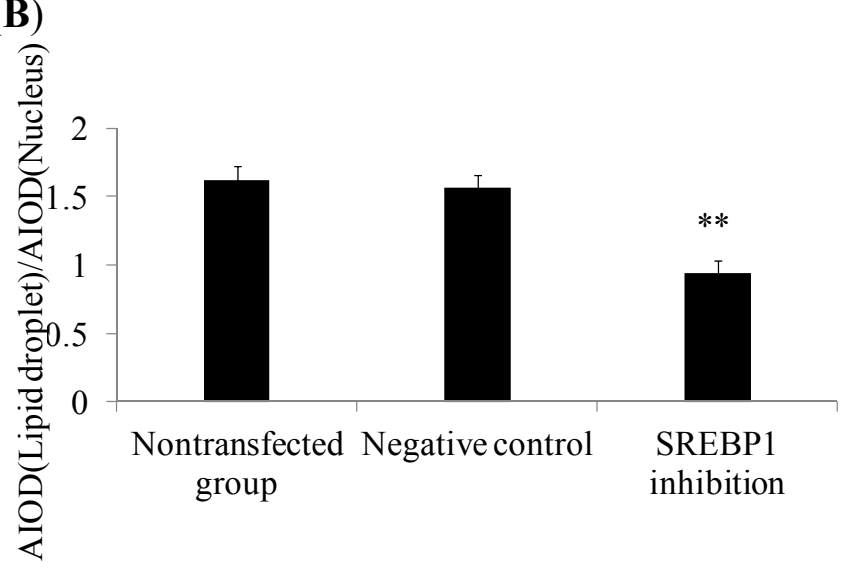

(D) 
2.3. The Effect of Stearic Acid and Serum on SREBPI and mTOR Expression in the Regulation of Milk Fat Synthesis

To determine the effect of stearic acid and serum on lipogenic gene expression in DCMECs, cells were treated with stearic acid $\left(100 \mu \mathrm{mol} \cdot \mathrm{L}^{-1}\right)$ and serum $(10 \% \mathrm{FBS})$, and were harvested at $24 \mathrm{~h}$ after treatment. The mRNA levels of SREBP1, ACC, FAS, SCD, PPAR , mTOR, and FABP3 were found to be up-regulated in cells treated with stearic acid $(D+S)$, in cells treated with FBS $(D+B)$, and in cells treated with stearic acid + FBS (D + S + B) compared with untreated (D) cells (Figure 4A). As shown by Western blotting analysis, the protein levels of SREBP1, p-SREBP1, mTOR, and p-mTOR were all increased in the $\mathrm{D}+\mathrm{S}, \mathrm{D}+\mathrm{B}$, and $\mathrm{D}+\mathrm{S}+\mathrm{B}$ groups compared with the $\mathrm{D}$ group (Figure 4B,C). Stearic acid treatment resulted in obvious notable increase in triglyceride secretion (Figure 4D) in DCMECs. These findings indicate that stearic acid and serum activate SREBP1 to enhance milk fat synthesis, and that the mTOR signaling pathway is involved in the observed effect.

Figure 4. The effect of stearic acid and serum on the regulation of milk fat synthesis by SREBP1. Four groups of DCMECs were assessed: Untreated cells grown in DF12 media (control group, D), DCMECs treated with stearic acid $(0.1 \mathrm{mM})+$ DF12 $(\mathrm{D}+\mathrm{S})$, DCMECs treated with DF12 and 10\% FBS $(\mathrm{D}+\mathrm{B})$, and DCMECs treated with stearic acid $(0.1 \mathrm{mM})+\mathrm{DF} 12+10 \%$ FBS $(\mathrm{D}+\mathrm{S}+\mathrm{B})$. In each group cells were treated for $24 \mathrm{~h}$. (A) Relative mRNA levels (gene of interest/ $\beta$-actin) of the indicated genes were determined by qRT-PCR; (B) The expression levels of the indicated proteins were assessed by Western blotting. P and $\mathrm{N}$ denote the precursor and cleaved nuclear forms of SREBP1, respectively;

(C) Quantification of protein of interest/ $\beta$-actin relative fold by gray scale scan; and (D) TG content in the culture supernatant of DCMECs. Values represent means $\pm \mathrm{SE}(n=3)$. $* p<0.05, * * p<0.01$ compared with negative control group.

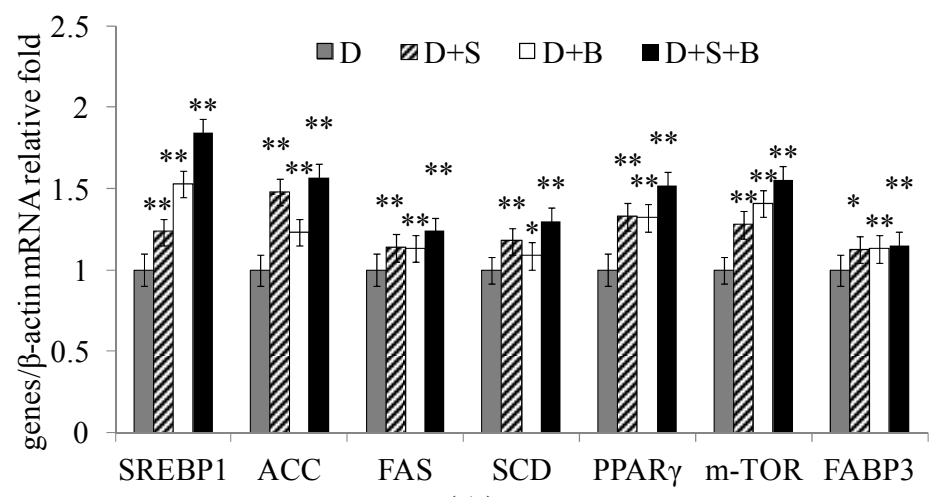

(A)

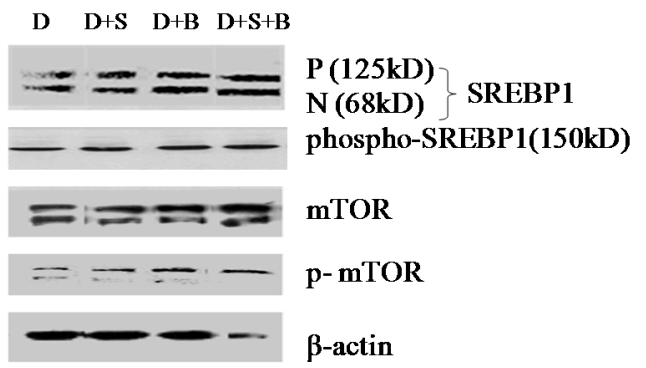

(B)

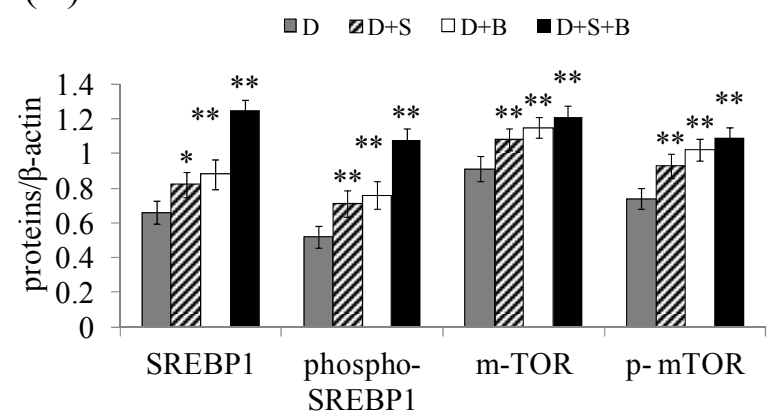

(C) 
Figure 4. Cont.

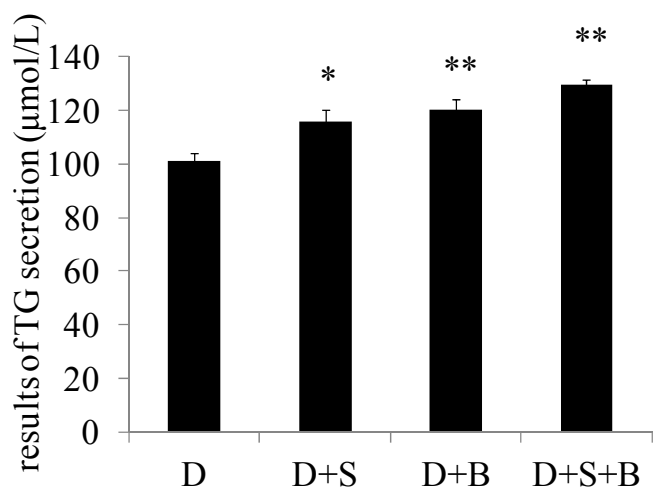

(D)

In agreement with the Western blotting results, the expression levels of SREBP1 and p-mTOR were also shown by immunofluorescence microscopy to be increased in response to stearic acid treatment (Figure 5A,B). Stearic acid treatment resulted in obvious notable increase in lipid droplet formation (Figure 5C,D) in DCMECs. These results are in accord with the aforementioned conclusion, further revealing the activities of SREBP1 and mTOR sense to stearic acid and serum to enhance milk fat synthesis.

Figure 5. The effect of stearic acid and serum on the subcellular localization of molecules related to milk fat synthesis. Groups of DCMECs were assessed as in Figure 4. (A) Localization of SREBP1 and p-mTOR. SREBP1 (green), giantin (purple), and DAPI (dark blue). Scale bars $=10 \mu \mathrm{m}$; (B) Integral optical density analysis of p-mTOR. p-mTOR (green), giantin (purple), and DAPI (blue). Scale bars $=10 \mu \mathrm{m}$; and $(\mathbf{C}, \mathbf{D})$ Immunofluorescent staining and integral optical density analysis of lipid droplets. Lipid droplet (green) and DAPI (blue). Scale bars $=10,15,45$ or $60 \mu \mathrm{m}$. Values represent means $\pm \mathrm{SE}(n=3) .{ }^{* *} p<0.01$ compared with negative control group.
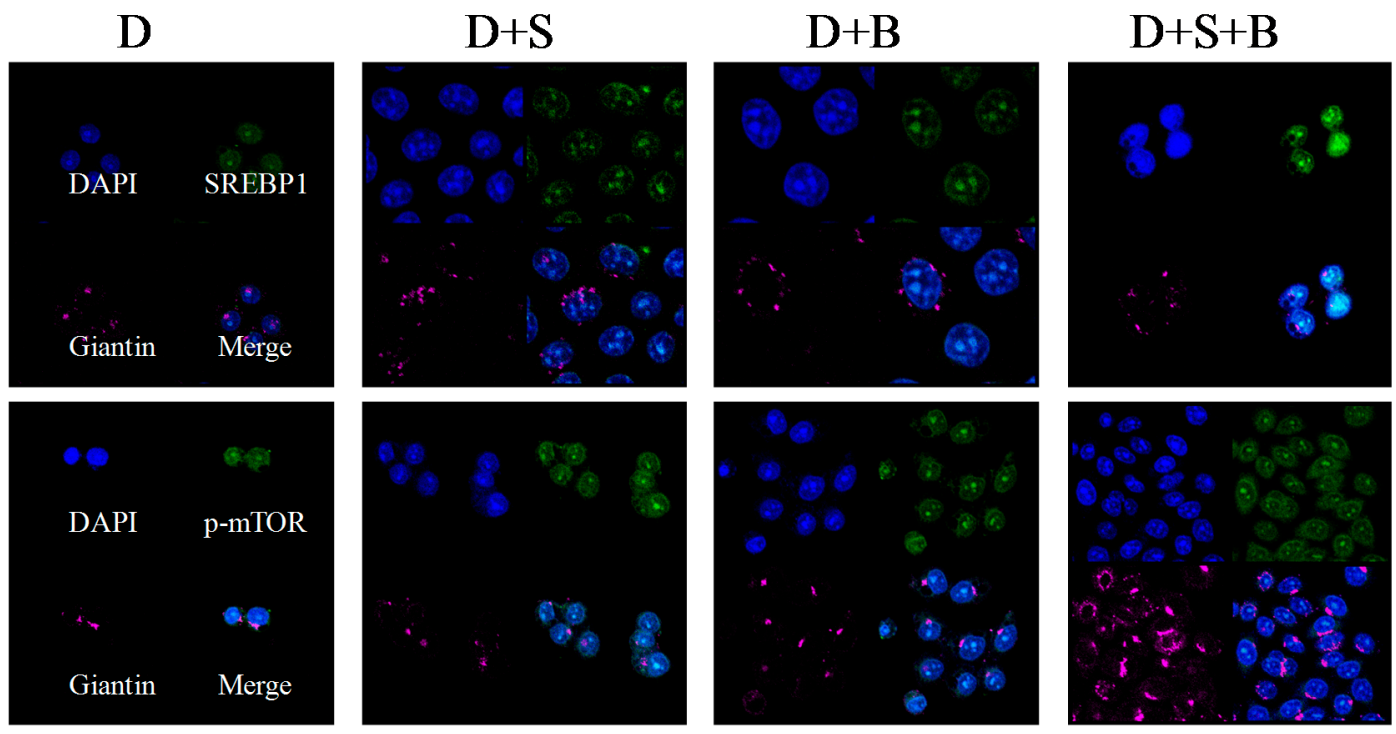

(A) 
Figure 5. Cont.
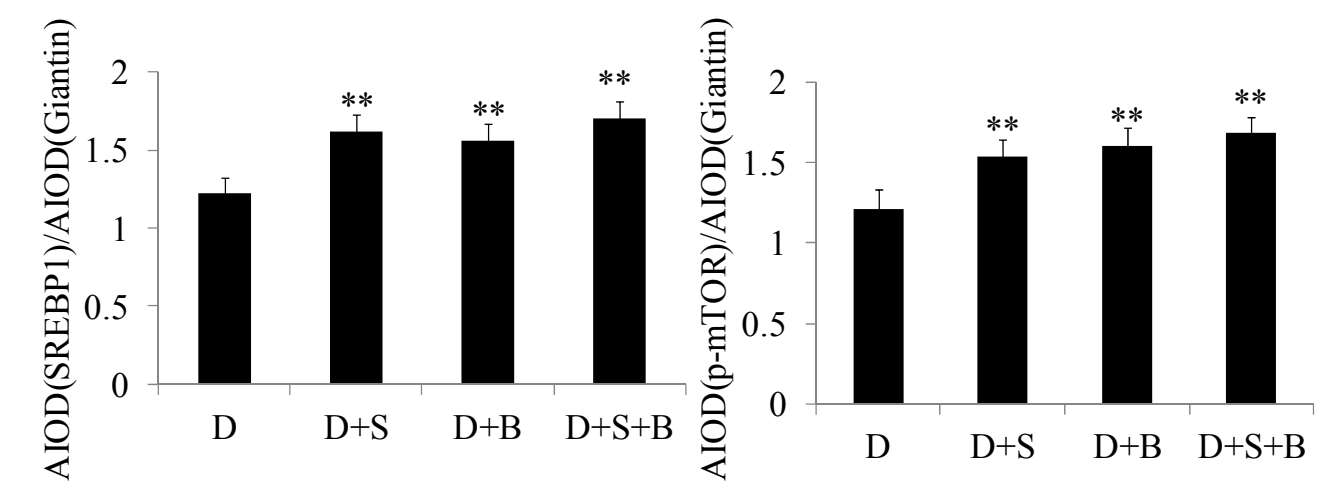

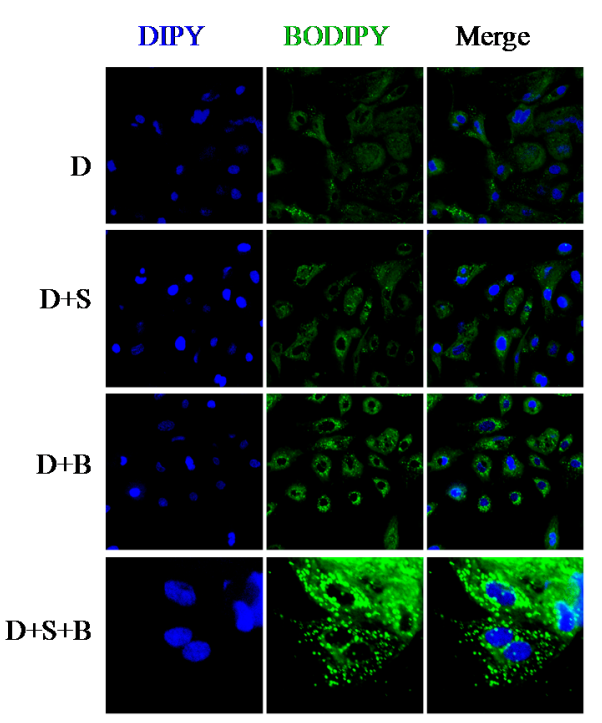

(C)
(B)

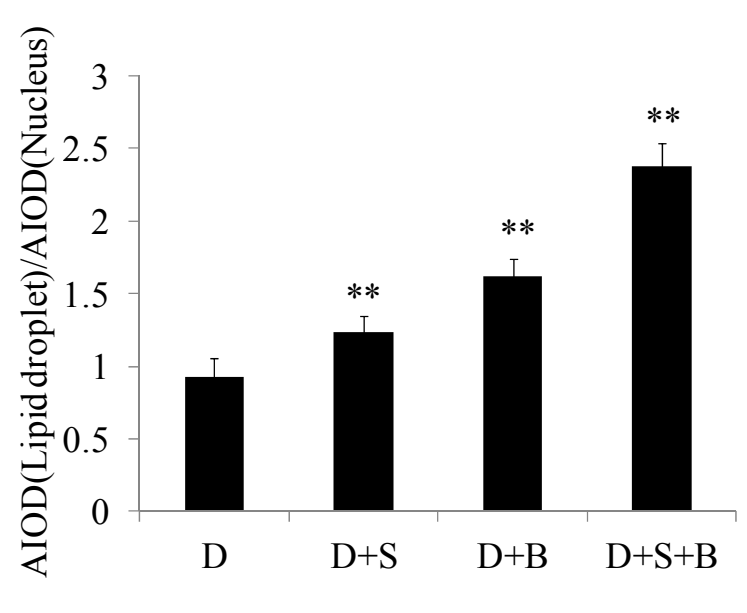

(D)

\subsection{Discussion}

The SREBP family of transcription factors is critical to the regulation of fatty acid and cholesterol biosynthetic gene expression [23]. Studies by Brown, Goldstein, and colleagues have elucidated key parts of the mechanism by which cholesterol sensing is coupled to the activity of SREBPs [24]. Few reports on phospho-SREBP1 (p-SREBP1) exist. It has been shown that SREBPs are inactive precursors and that the amino-terminal fragment of SREBP (nSREBP1c) is the active form of the protein and translocates to the nucleus. A newly generated p-SREBP1c-specific Ser372 antibody was used in this study. Li demonstrated that Ser372 phosphorylation of SREBP1c by AMPK (AMP-activated protein kinase) is necessary for inhibition of the proteolytic processing and transcriptional activity of SREBP1c. The phosphorylation of SREBP1c is an inactivation mechanism. AMPK stimulates Ser372 phosphorylation, suppresses SREBP1c cleavage and nuclear translocation, and represses SREBP1c target gene expression in hepatocytes exposed to high glucose, leading to reduced lipogenesis and lipid accumulation [25]. In this study, phosphorylated SREBP1 was found to be associated with increasing or decreasing SREBP1 expression. This may be interpreted as a dynamic balance between the nonphosphorylated, active form and the phosphorylated, inactive form.

Using SREBP1 overexpression and gene silencing experiments we have demonstrated that SREBP1 increases the relative mRNA level of FABP3. Fatty acid binding protein (FABP) and acyl-CoA binding 
protein (ACBP) are the main intracellular FA transporters in nonruminant cells [26]. The former has a high affinity for long-chain FA (LCFA) but also binds acyl-CoA [27,28]. In the current study, we confirm that SREBP1 regulates the expression of FABP3 to affect milk fat synthesis.

SREBP1 over-expression resulted in an increase in the mRNA levels of $A C C, F A S, S C D, m-T O R$, and FABP3 and a slight decrease in PPAR $\gamma$ mRNA, while SREBP1 gene silencing had the opposite effect. PPAR $\gamma$ is a member of the PPAR family of transcription factors and nuclear receptors and plays a pivotal part in cell fate determination, lipid biosynthesis, inflammation, and insulin sensitivity [29]. Recently reported findings suggest that the activation of PPAR $\gamma$ and PPAR $\gamma$ reduces triacylglycerol synthesis in rat hepatoma cells by reducing nuclear SREBP1 levels [30]. Our results further indicate that SREBP1 may be a negative regulator of PPAR $\gamma$ in DCMECs, and we speculate that PPAR $\gamma$ exercises its role in balancing milk fat synthesis.

Lipid synthesis involves the de novo synthesis of FA as well as the incorporation of de novo and preformed FA into triglycerides. The triglycerides accumulate to form lipid droplets, primarily in the liver, adipose, and mammary gland tissues of mammals. The activation of these metabolic pathways requires the coordinated regulation of a network of genes encoding lipogenic enzymes, such as the de novo FA synthesis genes $F A S$ and $A C C$, as well as the FA modification gene SCD. SREBP1 translocates to the nucleus where it activates lipogenic genes by binding to the SREBP1 response element of target genes, such as ACC and FAS [31]. The mRNA expression levels of $A C C, F A S$ and $S C D$, as well as triglyceride secretion and lipid droplets represent the cells' capacity for lipid synthesis. It was previously noted that SREBP1 activates ACC, FAS, and SCD [5], and in this study, we observed that SREBP1 affects the mRNA expression levels of the $A C C, F A S$, and $S C D$ genes in accordance with changes in triglyceride content and lipid droplet accumulation, further confirming that SREBP1 acts on its target genes to regulate milk fat synthesis in DCMECs.

In cells in which SREBP1 was over-expressed, the protein levels of m-TOR, and p-mTOR were elevated, whereas the opposite effects were observed when the SREBPl gene was silenced. Immunofluorescence was used to assess the localization of SREBP1 and p-mTOR, and revealed that SREBPI gene silencing reduces the activity of $\mathrm{p}-\mathrm{mTOR}$ protein in DCMECs. Our results suggest that the expression and subcellular localization of $\mathrm{p}-\mathrm{mTOR}$ are regulated by SREBP1. Considering that $\mathrm{p}-\mathrm{mTOR}$ regulates SREBP1 activity, our findings also suggest that a positive feedback loop may exist to regulate the SREBP1 and mTOR signaling pathways in DCMECs: SREBP1 over-expression promotes mTOR signaling in milk fat synthesis of DCMECs, whereas gene silencing of SREBPl weakens the mTOR signaling pathway.

LCFAs have been identified as an important cellular source of metabolic energy, as a substrate for membrane biogenesis (phospholipid), and for storage of metabolic energy (triglycerides and cholesterol esters) [26]. Serum and glucose or amino acid deprivation are known to strongly down-regulate SREBP target gene expression compared with cells grown in complete media or media lacking only serum [22]. We, thus, assessed the effect of stearic acid and serum on milk fat synthesis in DCMECs to ascertain whether SREBP1 is associated with the regulation of milk fat synthesis. Our experiments revealed that $A C C, F A S, S C D, P P A R \gamma, m-T O R$, and FABP3 mRNA levels were significantly increased by the addition of stearic acid and serum. Several LCFAs are natural ligands of non-ruminant PPAR $\gamma$, which, along with its lipogenic target genes, is up-regulated in bovine mammary tissue during lactation [32]. Unlike serum deprivation alone, serum deprivation combined with stearic 
acid deprivation was shown to down-regulate these genes. The protein levels of SREBP1, p-SREBP1, $\mathrm{m}$-TOR, and p-mTOR were shown to increase after the addition of stearic acid and serum, as were the triglyceride secretion and lipid droplet formation. From these findings, we conclude that fatty acids such as stearic acid and components in serum including lipogenic hormones activate the SREBP1 and mTOR signaling pathways to enhance milk fat synthesis in DCMECs.

\section{Experimental Section}

\subsection{Experimental Materials}

Instruments: Confocal microscope (TCS-SP2 AOBS, Leica, Wetzlar, Germany); ABI PRISM 7300 RT-PCR System (Applied Biosystems, Foster City, CA, USA).

Reagents: BODIPY 493/503 (Invitrogen, Carlsbad, CA, USA), Trizol (Invitrogen); Dulbecco's Modified Eagle medium-F12 (DF-12, Gibco, Carlsbad, CA, USA); fetal bovine serum (FBS, Gibco); insulin, prolactin, hydrocortisone and stearic acid (Sigma, MO, USA); nitrocellulose membrane (Bio-RAD, Shanghai, China); BCA Protein Quantitative Detection kit (GENMED Co., Ltd., Shanghai, China); monoclonal antibody for phospho-SREBP1, mTOR, p-mTOR (Cell Signaling Technology, Beverly, MA, USA); monoclonal antibody for SREBP1, giantin and $\beta$-actin (Santa Cruz Biotechnology Inc., Santa Cruz, CA, USA); HRP-conjugated anti-rabbit IgG, HRP-conjugated anti-goat IgG, HRP-conjugated anti-mouse IgG (ZSGB-BIO, Beijing, China); Super ECL Plus Detection Reagent (ApplyGEN, Beijing, China); triglyceride GPO-POD assay kit (ApplyGEN, Beijing, China).

\subsection{Primary DCMEC Culture and Treatment}

Primary DCMECs were cultured and purified according to previously reported methods [33,34]. Purified DCMECs were cultured in DF-12 media containing $10 \% \mathrm{FBS}$, insulin (bovine, $5 \mu \mathrm{g} \cdot \mathrm{mL}^{-1}$ ), hydrocortisone $\left(1 \mu \mathrm{g} \cdot \mathrm{mL}^{-1}\right)$, prolactin (bovine, $\left.5 \mu \mathrm{g} \cdot \mathrm{mL}^{-1}\right)$, penicillin $\left(100 \mathrm{U} \cdot \mathrm{mL}^{-1}\right)$, and streptomycin $\left(0.1 \mathrm{mg} \cdot \mathrm{mL}^{-1}\right)$. Before DCMEC treatment, culture medium was replaced with DF-12 media containing insulin, hydrocortisone, and prolactin (concentrations as above). For experimental assays, DCMECs in the logarithmic growth phase were plated at $3 \times 10^{4}$ cells $\cdot \mathrm{cm}^{-2}$.

\subsection{Gene Silencing of SREBP1}

DCMECs were transfected with SREBP1 siRNA or a negative scrambled control (GenePharma Co., Ltd., Shanghai, China) using Lipofectamine TM 2000 (LF2000, Invitrogen) according to the manufacturer's instructions. We screened siRNA-SREBP1a (sense5'-GAGGCCAAGUUGAAUAAA UTT-3'; antisense 5'-AUUUAUUCAACUUGGCCUCTT-3'), siRNA-SREBP1b (sense5'-GCUCCU CACUUGAAGGCUUTT-3'; antisense5'-AAGCCUUCAAGUGAGGAGCTT-3'), siRNA-SREBP1c (sense5'-GGAGGGUAUUCCUACAUGATT-3'; antisense5'-UCAUGUAGGAAUACCCUCCTT-3') and selected siRNA-SREBP1a for the highest gene silencing efficiency. The negative scrambled control siRNA (sense5'-UUCUCCGAACGUGUCACGUTT-3'; antisense5'-ACGUGACACGUUCG GAGAATT-3') had no significant homology to any gene of DCMECs. For silencing of SREBP1 gene expression, $1 \mu \mathrm{g}$ of siRNA and $2 \mu \mathrm{L}$ of LF2000 were diluted in $200 \mu \mathrm{L}$ of OPTI-MEMI medium, and 
added to the wells in 6-well plates to be transfected. Transfected cells were cultivated for $24 \mathrm{~h}$, after which they were collected for further experiments.

\section{4. $p G C M V-I R E S-E G F P-S R E B P 1$ Construction and Transfection}

Total RNA from cultured DCMECs was extracted using Trizol reagent and cDNA was synthesized. Bos taurus sterol regulatory element binding transcription factor 1 (SREBF1) mRNA (NM_001113302.1) is $3983 \mathrm{bp}$ in length and encodes 1146 amino acids, with the CDS (coding sequence ) region extending from 50 to $3490 \mathrm{bp}$. The S2P cleavage site on the human and bovine SREBP1 protein is accompanied by the amino acid recognition sequence DRSRLALC. The cleavage site is situated between the amino acids $\mathrm{L}$ and C [29]. In this study, the sequence base pairs 50 to 1467 of bovine SREBPI mRNA were therefore amplified. This sequence encodes the $N$-terminus of the protein, a transmembrane domain, which enters the nucleus and binds to SREs (sterol regulatory elements) within the promoters of target genes.

The resulting PCR product was inserted into the pMD18-T plasmid (TaKaRa, Dalian, China), after which the plasmid identity was confirmed by digestion with the restriction enzymes NheI and HindIII (TaKaRa, Dalian, China) and by DNA sequencing. The SREBPI gene was cloned into the pGCMV-IRES-EGFP vector (GenePharma Co., Ltd., Shanghai, China). The recombinant plasmids were assessed by digestion with NheI and HindIII. Primers were designed with particular restriction enzyme sites to allow for the complete coding region of SREBP1 to be cloned. Forward primer: 5'-CTAGCTAGCATGGACGAGCCACCCTTCAAC-3' (NheI); Reverse primer: 5'-CCCAAGCTTA GCGGCTCTGGATTCACCTG-3' (HindIII). For optimal amplification, annealing and extension steps were carried out at 55.7 and $72{ }^{\circ} \mathrm{C}$, respectively, for 35 cycles.

Transient transfections were carried out as previously described by Lu [35]. Briefly, DCMECs were transfected with pGCMV-IRES-EGFP-SREBP1 or with an empty vector using Lipofectamine TM 2000 (LF2000, Invitrogen, Camarillo, CA, USA) according to the manufacturer's instructions. Nontransfected cells were used as controls. Cells were cultivated for $24 \mathrm{~h}$, after which they were collected for further experiments.

\subsection{BODIPY Staining of Lipid Droplets}

Cells were washed with PBS, fixed with 3\% formaldehyde for $15 \mathrm{~min}$, and stained with BODIPY 493/503 (Invitrogen, stock concentration $1 \mathrm{mg} / \mathrm{mL}$, working solution 1:1000 dilution) for $15 \mathrm{~min}$ at room temperature. Cells were then mounted with Prolong gold anti-fade reagent (Invitrogen) followed by three washes in phosphate buffered saline (PBS) [36].

\subsection{Detection of Triglyceride Secretion}

Cell-free supernatants were employed in triglyceride quantification using the TG GPO-POD Assay kit (Applygen Tech Inc., Beijing, China) according to the manufacturer's instructions.

\subsection{RNA Extraction and Real-Time Quantitative PCR}

Total RNA from DCMECs was extracted using Trizol reagent according to the manufacturer's instructions. Total RNA ( $1 \mu \mathrm{g})$ was transcribed into cDNA using Thermoscript Reverse Transcriptase 
(TaKaRa, Dalian, China) according to the manufacturer's instructions. After reverse transcription, PCR reactions were carried out using an ABI PRISM 7300 RT-PCR System. $\beta$-actin was used as a reference gene. The primers of these gene transcripts were as follows: SREBP1: sense5'-ACGCCAT CGAGAAACGCTAC-3', antisense5'-GTGCGCAGACTCAGGTTCTC-3'; ACC: sense5'-GGGTGAAA GACTGGGTTGAA-3', antisense5'-GACAGAGCACGGATGTGATG-3'; FAS: sense5'-CTGCAAC TCAACGGGAACTT-3', antisense5'-AGGCTGGTCATGTTCTCCAG-3'; SCD: sense5'-CCCTTTCC TTGAGCTGTCTG-3', antisense5'-ATGCTGACTCTCTCCCCTGA-3'; PPAR $\gamma$ : sense5'-TCAAAG TGGAGCCTGTATC-3', antisense5'-CATAGTGGAACCCTGACG-3'; FABP3: sense5'-GAACTCGAC TCCCAGCTTGAA-3', antisense5'-AAGCCTACCACAATCATCGAAG-3'; mTOR: sense5'-ATGCT GTCCCTGGTCCTTATG-3', antisense5'-GGGTCAGAGAGTGGCCTTCAA-3'; $\beta$-actin: sense5'-AAG GACCTCTACGCCAACACG-3', antisense5'-TTTGCGGTGGACGATGGAG-3'. RT-PCR analysis was performed using the $\Delta \Delta C_{\mathrm{t}}$ method [37].

\subsection{Immunofluorescence Assay}

Immunofluorescence assays were performed using standard techniques [13] to assess SREBP1 and p-mTOR localization.

\subsection{Western Blotting Analysis}

Western blotting analysis was performed using standard techniques [12] to detect the expression of SREBP1, p-SREBP1, mTOR, p-mTOR, and $\beta$-actin.

\subsection{Bioinformatics Analysis}

The mRNA sequence of Bos SREBP1 was sourced from the NCBI nucleotide database (http://www.ncbi.nlm.nih.gov/nuccore) and the SMART online tool (http://smart.embl-heidelberg.de) was used to analyze the structure and function of the SREBP1 protein.

\subsection{Statistical Analysis}

Results were reported as mean \pm SE. Data statistics and individual differences among groups were analyzed by using IBM SPSS Statistics 21 software (IBM, Armonk, New York, NY, USA). A statistical comparison of the means among the groups was performed using one-way analysis of variance. Differences between the means of individual groups were analyzed by Tukey post hoc tests. Differences with $p<0.05$ were considered statistically significant and those with $p<0.01$ were considered extremely significant. The integral optical densities of laser confocal images were analyzed using Image-Pro Plus 6.0 software (Media Cybernetics, Inc., Rockville, MD, USA) and the gray-scale scanning of Western blots was analyzed using Glyko Band Scan 5.0 software (Glyko, Hayward, CA, USA).

\section{Conclusions}

Together, these results reveal that SREBP1 is a positive regulator to promote milk fat synthesis in DCMECs. These data also suggest that there is a positive feedback-loop regulation between SREBP1 and mTOR signaling pathways in DCMECs. SREBP1 promotes the mTOR signaling pathway and 
milk fat synthesis of DCMECs. In the other hand, stearic acid and serum can up-regulate SREBP1 to enhance milk fat synthesis.

\section{Acknowledgments}

This study was financially supported by the Major State Basic Research Development Program of China (973 Program, No. 2011CB100804) and the High Technology Project of the Ministry of Science and Technology of China (863 Project, No. 2013AA102504-03).

\section{Author Contributions}

Nan Li developed the conception and design of the study, analyzed data and co-drafting of the manuscript; Feng Zhao performed immunofluorescence experimental work and co-drafting of the manuscript; Chenjie Wei performed quantitation of secreted triglyceride and immunofluorescence experimental work; Mengyao Liang co-drafting of the manuscript; Na Zhang participated in collection of the literature review; Chunmei Wang analyzed data; Qing-zhang Li analyzed data and revision of the manuscript; and Xue-jun Gao suggested the research idea, supervised the project and revision of the manuscript.

\section{Conflicts of Interest}

The authors declare no conflict of interest.

\section{References}

1. Hua, X.; Wu, J.; Goldstein, J.L.; Brown, M.S.; Hobbs, H.H. Structure of the human gene encoding sterol regulatoryelement binding protein 1 (SREBF1) and localization of SREBF1 and SREBF2 to chromosomes 17p11.2 and 22q13. Genomics 1995, 25, 667-673.

2. Yokoyama, C.; Wang, X.; Briggs, M.R.; Admon, A.; Wu, J.; Hua, X.; Goldstein, J.L.; Brown, M.S. SREBP1, a basic helixloop-helix leucine zipper protein that controls transcription of the LDL receptor gene. Cell 1993, 75, 187-197.

3. Sato, R.; Yang, J.; Wang, X.; Evans, M.J.; Ho, Y.K.; Goldstein, J.L.; Brown, M.S. Assignment of the membrane attachment, DNA binding, and transcriptional activation domains of sterol regulatory element binding protein 1 (SREBP1). J. Biol. Chem. 1994, 269, 17267-17273.

4. Naar, A.M.; Beaurang, P.A.; Robinson, K.M.; Oliner, J.D.; Avizonis, D.; Scheek, S.; Zwicker, J.; Kadonaga, J.T.; Tjian, R. Chromatin, TAFs, and a novel multiprotein coactivator are required for synergistic activation by Sp1 and SREBP1a in vitro. Genes Dev. 1998, 12, 3020-3031.

5. McPherson, R.; Gauthier, A. Molecular regulation of SREBP function: The Insig-SCAP connection and isoform-specific modulation of lipid synthesis. Biochem. Cell Biol. 2004, 2, 201-211.

6. Bennett, M.K.; Toth, J.I.; Osborne, T.F. Selective association of sterol regulatory element-binding protein isoforms with target promoters in vivo. J. Biol. Chem. 2004, 279, 37360-37367.

7. Llorente-Cortés, V.; Costales, P.; Bernués, J.; Camino-Lopez, S.; Badimon, L. Sterol regulatory element-binding protein-2 negatively regulates low density lipoprotein receptor-related protein transcription. J. Mol. Biol. 2006, 359, 950-960. 
8. Robichon, C.; Varret, M.; Le, L.X.; Lasnier, F.; Hajduch, E.; Ferré, P.; Dugail, I. DnaJA4 is a SREBP-regulated chaperone involved in the cholesterol biosynthesis pathway. Biochim. Biophys. Acta 2006, 1761, 1107-1113.

9. Laaksonen, R.; Thelen, K.M.; Paiva, H.; Matinheikki, J.; Vesalainen, R.; Janatuinen, T.; Knuuti, J.; Rontu, R.; von Bergmann, K.; Lütjohann, D.; et al. Genetic variant ofthe SREBF1 gene is significantly related to cholesterol synthesis in man. Atherosclerosis 2006, 185, 206-209.

10. Ma, L.; Corl, B.A. Transcriptional regulation of lipid synthesis in bovine mammary epithelial cells by sterol regulatory element binding protein 1. J. Dairy Sci. 2012, 95, 3743-3755.

11. Bionaz, M.; Loor, J.J. Gene networks driving bovine milk fat synthesis during the lactation cycle. BMC Genomics 2008, 9, 366.

12. Wan, Z.Y.; Tong, H.L.; Li, Q.Z.; Gao, X.J. Influence on cellular signal transduction pathway in dairy cow mammary gland epithelial cells by galactopoietic compound isolated from Vaccariae segetalis. Agric. Sci. China 2011, 10, 101-105.

13. Huang, Y.L.; Zhao, F.; Luo, C.C.; Zhang, X.; Si, Y.; Sun, Z.; Zhang, L.; Li, Q.Z.; Gao, X.J. SOCS3-mediated blockade reveals major contribution of JAK2/STAT5 signaling pathway to lactation and proliferation of dairy cow mammary epithelial cells in vitro. Molecules 2013, 18, 12987-13000.

14. Liu, G.; Zhang, Y.; Xu, D.; Zhang, R.; Cheng, Y.; Pan, Y.; Huang, C.; Chen, Y. Luteolin alleviates alcoholic liver disease induced by chronic and binge ethanol feeding in mice. J. Nutr. 2014, doi:10.3945/jn.114.193128.

15. Bakan, I.; Laplante, M. Connecting mTORC1 signaling to SREBP1 activation. Curr. Opin. Lipidol. 2012, 23, 226-234.

16. Yecies, J.L.; Zhang, H.H.; Menon, S.; Liu, S.; Yecies, D.; Lipovsky, A.I.; Gorgun, C.; Kwiatkowski, D.J.; Hotamisligil, G.S.; Lee, C.H. et al. Akt stimulates hepatic SREBP1c and lipogenesis through parallel mTORC1-dependent and independent pathways. Cell Metab. 2011, 14, 21-32.

17. Li, S.; Ogawa, W.; Emi, A.; Hayashi, K.; Senga, Y.; Nomura, K.; Hara, K.; Yu, D.; Kasuga, M. Role of S6K1 in regulation of SREBP1c expression in the liver. Biochem. Biophys. Res. Commun. 2011, 412, 197-202.

18. Li, S.; Brown, M.S.; Goldstein, J.L. Bifurcation of insulin signaling pathway in rat liver: mTORC1 required for stimulation of lipogenesis, but not inhibition of gluconeogenesis. Proc. Natl. Acad. Sci. USA 2010, 107, 3441-3446.

19. Dü vel, K.; Yecies, J.L.; Menon, S.; Raman, P.; Lipovsky, A.I.; Souza, A.L.; Triantafellow, E.; Ma, Q.; Gorski, R.; Cleaver, S.; et al. Activation of a metabolic gene regulatory network downstream of mTOR complex 1. Mol. Cell. 2010, 39, 171-183.

20. Porstmann, T.; Santos, C.R.; Griffiths, B.; Cully, M.; Wu, M.; Leevers, S.; Griffiths, J.R.; Chung, Y.L.; Schulze, A. SREBP activity is regulatedby mTORC1 and contributes to Akt-dependent cell growth. Cell Metab. 2008, 8, 224-236.

21. Moule, S.K.; Edgell, N.J.; Welsh, G.I.; Diggle, T.A.; Foulstone, E.J.; Heesom, K.J.; Proud, C.G.; Denton, R.M. Multiple signalling pathways involved in the stimulation of fatty acid and glycogen synthesis by insulin in rat epididymal fat cells. Biochem. J. 1995, 311, 595-601.

22. Peterson, T.R.; Sengupta, S.S.; Harris, T.E.; Carmack, A.E.; Kang, S.A.; Balderas, E.; Guertin, D.A.; Madden, K.L.; Carpenter, A.E.; Finck, B.N.; et al. mTOR complex 1 regulates lipin 1 localization to control the SREBP pathway. Cell 2011, 146, 408-420. 
23. Horton, J.D.; Goldstein, J.L.; Brown, M.S. SREBPs: Activatorsof the complete program of cholesterol and fatty acid synthesis in the liver. J. Clin. Investig. 2002, 109, 1125-1131.

24. Brown, M.S.; Goldstein, J.L. Cholesterol feedback: From Schoenheimer's bottle to Scap's MELADL. J. Lipid Res. Suppl. 2009, 50, S15-S27.

25. Li, Y.; Xu, S.; Mihaylova, M.M.; Zheng, B.; Hou, X.; Jiang, B.; Park, O.; Luo, Z.; Lefai, E.; Shyy, J.Y.; et al. AMPK phosphorylates and inhibits SREBP activity to attenuate hepatic steatosis and atherosclerosis in diet-induced insulin-resistant mice. Cell Metab. 2011, 13, 376-388.

26. McArthur, M.J.; Atshaves, B.P.; Frolov, A.; Foxworth, W.D.; Kier, A.B.; Schroeder, F. Cellular uptake and intracellular trafficking of long chain fatty acids. J. Lipid Res. 1999, 40, 1371-1383.

27. Frolov, A.; Cho, T.H.; Murphy, E.J.; Schroeder, F. Isoforms of rat liver fatty acid binding protein differ in structure and affinity forfatty acids and fatty acyl CoAs. Biochemistry 1997, 36, 6545-6555.

28. Whetstone, H.D.; Hurley, W.L.; Davis C.L. Identification and characterization of a fatty acid binding protein in bovine mammary gland. Comp. Biochem. Physiol. B 1986, 85, 687-692.

29. Kawaiand, M.; Rosen, C.J. PPAR $\gamma$ : A circadian transcription factor in adipogenesis and osteogenesis. Nat. Rev. Endocrinol. 2010, 6, 629-636.

30. König, B.; Koch, A.; Spielmann, J.; Hilgenfeld, C.; Hirche, F.; Stangl, G.I.; Eder, K. Activation of PPAR $\alpha$ and PPAR $\gamma$ reduces triacylglycerol synthesis in rat hepatoma cells by reduction of nuclear SREBP1. Eur. J. Pharmacol. 2009, 605, 23-30.

31. Kim, Y.M.; Shin, H.T.; Seo, Y.H.; Byun, H.O.; Yoon, S.H.; Lee, I.K.; Hyun, D.H.; Chung, H.Y.; Yoon, G. Sterol regulatory element-binding protein (SREBP) 1 mediated lipogenesis is involved in cell senescence. J. Biol. Chem. 2010, 285, 29069-29077.

32. Kadegowda, A.K.; Bionaz, M.; Piperova, L.S.; Erdman, R.A.; Loor, J.J. Peroxisome proliferator-activated receptor- $\gamma$ activation and long-chain fatty acids alter lipogenic gene networks in bovine mammary epithelial cells to various extents. J. Dairy Sci. 2009, 92, 4276-4289.

33. Tong, H.-L.; Gao, X.-J.; Li, Q.-Z.; Liu, J.; Li, N.; Wan, Z.-Y. Metabolic regulation of mammary gland epithelial cells of dairy cow by galactopoietic compound isolated from Vaccariae segetalis. Agric. Sci. China 2011, 10, 1106-1116.

34. Lu, L.-M; Gao, X.-J; Li, Q.-Z; Huang, J.-G; Liu, R.; Li, H. Comparative phosphoproteomics analysis of the effects of L-methionine on dairy cow mammary epithelial cells. Can. J. Anim. Sci. 2012, 92, 433-442.

35. Lu, L.M.; Li, Q.Z.; Huang, J.G.; Gao, X.J. Proteomic and functional analyses reveal MAPK1 regulates milk protein synthesis. Molecules 2013, 18, 263-275.

36. Lee, J.S.; Mendez, R.; Heng, H.H.; Yang, Z.Q.; Zhang, K. Pharmacological ER stress promotes hepatic lipogenesis and lipid droplet formation. Am. J. Transl. Res. 2012, 4, 102-113.

37. Huang, J.G.; Gao, X.J.; Li, Q.Z.; Lu, L.M.; Liu, R.; Luo, C.C.; Wang, J.L.; Qiao, B.; Jin, X. Proteomic analysis of the nuclear phosphorylated proteins in dairy cowmammary epithelial cells treated with estrogen. Vitro Cell. Dev. Biol. Anim. 2012, 48, 449-457.

(C) 2014 by the authors; licensee MDPI, Basel, Switzerland. This article is an open access article distributed under the terms and conditions of the Creative Commons Attribution license (http://creativecommons.org/licenses/by/3.0/). 